\title{
The role of virtual-assisted lung mapping 2.0 combining microcoils and dye marks in deep lung resection
}

Masaaki Sato, MD, PhD, ${ }^{\text {a }}$ Masashi Kobayashi, MD, PhD, ${ }^{\mathrm{b}}$ Jin Sakamoto, MD, PhD, ${ }^{\mathrm{c}}$ Ryuta Fukai, MD, PhD, ${ }^{d}$ Hiromitsu Takizawa, MD, PhD, ${ }^{e}$ Shinji Shinohara, MD, PhD, ${ }^{f}$ Fumitsugu Kojima, MD, $\mathrm{PhD},{ }^{\mathrm{g}}$ Akira Sakurada, $\mathrm{MD}, \mathrm{PhD},{ }^{\mathrm{h}}$ and Jun Nakajima, $\mathrm{MD}, \mathrm{PhD}^{\mathrm{a}}$

\section{ABSTRACT}

Objectives: Virtual-assisted lung mapping 2.0 is a novel preoperative bronchoscopic lung mapping technique combining the multiple dye marks of conventional virtual-assisted lung mapping with intrabronchial microcoils to navigate thoracoscopic deep lung resection. This study's purpose was to evaluate the feasibility of virtual-assisted lung mapping 2.0 in resecting deeply located pulmonary nodules with adequate margins.

Methods: A multicenter, prospective single-arm study was performed from 2019 to 2020 in 8 institutions. The selection criteria were barely identifiable nodules requiring sublobar lung resections, nodules requiring resection lines reaching the inner $2 / 3$ of the pulmonary lobe on computed tomography images in wedge resection, or the nodule center located in the inner $2 / 3$ of the pulmonary lobe in wedge resection or segmentectomy. Resection margins larger than $2 \mathrm{~cm}$ or the nodule diameter were considered successful resection. Bronchoscopic placement of multiple dye marks and microcoil(s) was conducted o to 2 days before surgery.

Results: We analyzed 65 lesions in 64 patients. The diameter and depth of the targeted nodules and the minimum required resection depth reported as median (interquartile range) were 9 (7-13) mm, 11 (5-15) $\mathrm{mm}$, and 30 (25-35) mm, respectively. Among 60 wedge resections and 5 segmentectomies, successful resection was achieved in 64 of 65 resections ( $98.5 \%$; 95\% confidence interval, 91.7-100). Among 75 microcoils placed, 3 showed major displacement after bronchoscopic placement. There were no severe adverse events associated with the virtual-assisted lung mapping procedure.

Conclusions: This study demonstrated that virtual-assisted lung mapping 2.0 can facilitate successful resections for deep pulmonary nodules, overcoming the limitations of conventional virtual-assisted lung mapping. (J Thorac Cardiovasc Surg 2022;164:243-51)

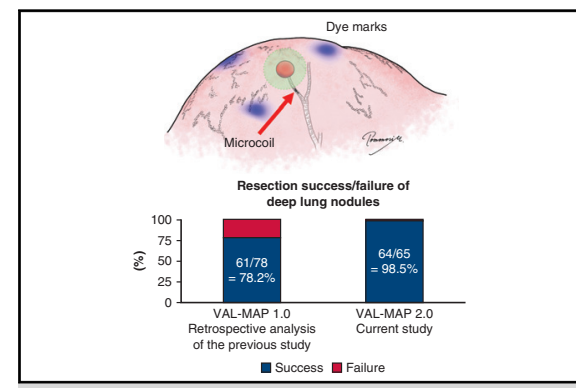

VAL-MAP 2.0 (dye + microcoil) secured deep resection margins in sublobar lung resections.

CENTRAL MESSAGE

VAL-MAP 2.0, combining preoperative bronchoscopic placement of dye marks and microcoils, enabled acquisition of secure resection margins in sublobar lung resection of deep tumors.

\section{PERSPECTIVE}

This multicenter prospective trial demonstrated that VAL-MAP 2.O, combining bronchoscopic preoperative placement of multi-spot dye marks on the lung surface and microcoils in airways, enabled effective acquisition of secure resection margins in sublobar lung resections for deep tumors, overcoming the limitation of conventional VAL-MAP using dye marks alone.

See Commentary on page 252.

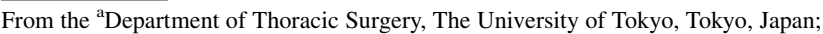
bepartment of Thoracic Surgery, Tokyo Medical and Dental University, Tokyo, Japan; ${ }^{\mathrm{C}}$ Department of Thoracic Surgery, Shimane Prefectural Central Hospital, Izumo, Japan; ${ }^{\mathrm{d}}$ Department of Thoracic Surgery, Shonan Kamakura General Hospital, Kamakura, Japan; ${ }^{\mathrm{e}}$ Department of Thoracic, Endocrine Surgery, and Oncology, Tokushima University Graduate School of Biomedical Sciences, Tokushima, Japan; ${ }^{\mathrm{f}}$ The Second Department of Surgery, University of Occupational and Environmental Health, Kitakyushu, Japan; ${ }^{\mathrm{g}}$ Department of Thoracic Surgery, St Luke's International Hospital, Tokyo, Japan; and ${ }^{\mathrm{h}}$ Department of Thoracic Surgery, Institute of Development, Aging and Cancer, Tohoku University, Sendai, Japan

This study was funded by the Japan Agency for Medical Research and Development. The clinical trial was registered at Japan Registry of Clinical Trials (Registry Number: jRCTs031180099). This study was approved by The University of Tokyo, Clinical Research Review Board on 1 October 2018 (2018003SP) for all institutes.
}

The entire study was approved as an Advanced Medical Care Program type B by the Advanced Medical Technology Review Board and Advanced Medical Treatment Committee (December 6, 2018) of the Japanese Ministry of Health, Labour, and Welfare. Written informed consent was obtained from all the patients at their registration.

Received for publication April 29, 2021; revisions received Aug 4, 2021; accepted for publication Sept 8, 2021; available ahead of print Sept 17, 2021.

Address for reprints: Masaaki Sato, MD, PhD, Department of Thoracic Surgery, The University of Tokyo Hospital, 7-3-1 Hongo, Bunkyo-ku, Tokyo 113-8655, Japan (E-mail: satom-sur@h.u-tokyo.ac.jp).

0022-5223

Copyright $(2021$ The Authors. Published by Elsevier Inc. on behalf of The American Association for Thoracic Surgery. This is an open access article under the CC BY-NC-ND license (http://creativecommons.org/licenses/by-nc-nd/4.0/).

https://doi.org/10.1016/j.jtcvs.2021.09.016 


\section{Abbreviations and Acronyms}

$\mathrm{CI}=$ confidence interval

CT $=$ computed tomography

VATS $=$ video-assisted thoracic surgery

VAL-MAP $=$ virtual-assisted lung mapping

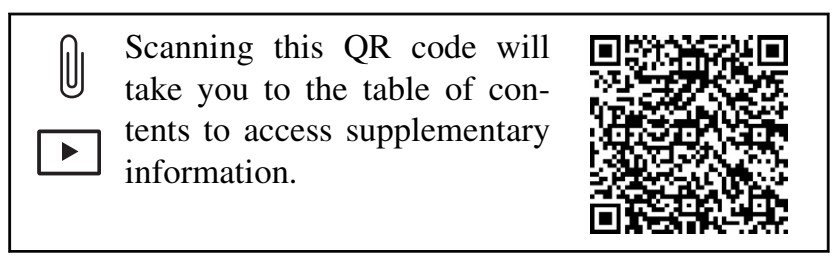

Localizing small pulmonary nodules, including ground-glass nodules (GGNs), is often challenging intraoperatively, especially in sublobar lung resection (wedge resection and segmentectomy). Among localization techniques, virtual-assisted lung mapping (VAL-MAP), a bronchoscopic preoperative multi-spot dye marking technique, ${ }^{1}$ is highly safe and reproducible. ${ }^{2-5}$ Notably, the multiple dye marks of VAL-MAP are designed to indicate sufficient resection margins, ${ }^{1,6}$ which have a significant impact on local recurrence and patient survival in sublobar lung resection. ${ }^{7,8}$ However, because the information provided by the dye marks is limited to the pleural surface, acquiring deep resection margins is a major concern. ${ }^{4}$ In a previous prospective study defining necessary resection margins as larger than or equal to the tumor diameter or $2 \mathrm{~cm}$, resection margins were insufficient in approximately $10 \%$ of the cases in which the depth of the required resection line reached $3 \mathrm{~cm} .{ }^{4}$ Another limitation of original VAL-MAP was the visibility of the blue dye in cases with prominent anthracosis or pleural adhesion. ${ }^{6}$ These limitations appear to be common among localization techniques using pleural markings. ${ }^{9}$

To overcome the challenges, we developed VAL-MAP 2.0, which combines bronchoscopic multi-spot dye marking and concurrent bronchoscopic microcoil placement in a distal bronchus. ${ }^{10}$ In addition to the 2-dimensional pleural dye marks of conventional VAL-MAP, the "z-axis" provided by an intrabronchial platinum microcoil realizes 3 -dimensional lung mapping. ${ }^{10}$ The microcoil is visualized intraoperatively using fluoroscopy to confirm deep resection margins. In our pilot study, this technique was found safe and feasible, with high potential to support satisfactory deep lung resection. The purpose of this study was to further evaluate the feasibility of VAL-MAP 2.0 in resecting deeply located pulmonary nodules with sufficient margins.

\section{MATERIALS AND METHODS}

This multicenter prospective single-arm study was conducted from February 2019 to June 2020 in 8 registered participating hospitals in Japan. The study was approved by The University of Tokyo, Clinical Research Review Board (Approval Number 218003SP) for all institutes, and the study was preregistered in the Japan Registry of Clinical Trials (jRCTs031180099). The study protocol proposal was published previously ${ }^{11}$ (Online Data Supplement).

\section{Patient Registration}

After obtaining written informed consent, patients meeting the inclusion criteria (Table 1) were enrolled. Briefly, resection of lesions with margins larger than or equal to the lesion diameter or $2 \mathrm{~cm}$ using planned sublobar resection (ie, wedge resection or segmentectomy) with curative intent was the primary requisite. In addition to the selection criteria for original VAL-MAP $^{1,4}$ (ie, barely identifiable nodules requiring sublobar lung resection), we included nodules requiring a resection line reaching the inner $2 / 3$ of the pulmonary lobe on computed tomography (CT) images in wedge resection or the nodule center located in the inner $2 / 3$ of the pulmonary lobe on $\mathrm{CT}$ images in segmentectomy or wedge resection (Figure E1). We also included cases wherein identifying both nodules and dye marks was expected to be difficult owing to pneumoconiosis, fibrosis, or other conditions.

\section{Virtual-Assisted Lung Mapping 2.0 Procedure}

The VAL-MAP 2.0 procedure was described previously ${ }^{10}$ (Figure 1, Video 1, Table E1). A 3-dimensional lung map was designed by translating thin-slice CT images into 3-dimensional images (Figure 1, A) using a radiology workstation (Synapse Vincent; FujiFilm Medical Inc, Tokyo, Japan), and virtual bronchoscopic images for each target mark (either dye mark or microcoil) were built. Bronchial roots to inject dye were designed to comprise 3 to 5 marks to indicate satisfactory resection margins on the pleural surface. A bronchial root for microcoil placement was determined according to the following principles to indicate the most important resection margins inside the lung: In wedge resection, if a bronchus directly reached the target lesion or was near the lesion, we selected the point of the bronchus central to the tumor to indicate the deep resection margin (Figure 2, A). If no bronchus directly reached the lesion, 2 bronchi reaching relatively close to the tumor were selected for microcoil placement (Figure 2, B). In segmentectomy, a bronchial root or roots were selected close to an intersegmental plane, where the resection margin would be most critical (Figure 2, C). Notably, although the deepest resection margin in segmentectomy is the hilum, the resection margin closest to the tumor is often on the intersegmental plane in a coneshaped resection in segmentectomy., ${ }^{2,12}$ To accurately place a microcoil through a bronchial root, the distance from a bronchial branching to the planned microcoil location was measured (eg, $22 \mathrm{~mm}$ from the seventh branching and $34 \mathrm{~mm}$ from the sixth branching) on virtual bronchoscopy.

\section{Mapping Procedure and Post-Mapping Computed Tomography}

Mapping was conducted on the day of surgery, or 1 or 2 days preoperatively depending on the center's logistics, with local or general anesthesia. First, multiple dye marks were made bronchoscopically using a metal blunt-tipped catheter (P6-CW-1; Olympus, Tokyo, Japan) preloaded with $1 \mathrm{~mL}$ per mark of indigo carmine, followed by bronchoscopic placement of a platinum C-stopper microcoil (final diameter, $5 \mathrm{~mm}$; Piolax Medical Devices, Tokyo, Japan). A microcoil was pushed through the microcatheter into the bronchial lumen under fluoroscopy, and the microcoil was automatically rolled and fixed against the bronchial wall ${ }^{10}$ (Figure 1, B). When the procedure was conducted with local anesthesia, the patient was awakened, and CT was performed to confirm the locations of the 
TABLE 1. Inclusion criteria

1 Patients with suspected/diagnosed pulmonary malignancy who require the establishment of resection lines other than the typical interlobar fissure

2 A tumor that is expected to be barely identifiable intraoperatively, and will thus need particularly accurate demarcation for visualization (indications for dye injection) for 1 of the following reasons:

Lesion characteristics

Lesions wholly or partially composed of ground-glass opacity

Lesions with a tumor diameter $\leq 5 \mathrm{~mm}$

Lesions with a distance from the visceral pleura larger than the tumor diameter

Underlying lung conditions

Severe advanced pleural adhesions (eg, history of open chest surgery)

Preexisting benign nodules that are confusing and misleading

3 A tumor that is expected to be barely identifiable intraoperatively with dye injection alone and is therefore expected to require the introduction of a microcoil, including any 1 of the following conditions:

A. Lesions indicated for wedge resection, with the margin on the central side expected to be barely determinable without microcoil application; that is, the resection line will reach the inner $2 / 3$ of the pulmonary lobe, but the tumor center is located in the outer $1 / 3$ of the pulmonary lobe on CT images*

B. Lesions indicated for sublobar resection (wedge resection or segmentectomy), with the margin on the central side expected to be barely determinable without microcoil placement; that is, the tumor center is located in the inner $2 / 3$ of the pulmonary lobe on CT images*

C. Anticipation of a substantial degree of lung pigmentation (eg, pneumoconiosis, emphysema, or fibrosis)

4 The patient has provided informed consent.

$C T$, Computed tomography. *The conceptual separation of the pulmonary lobe into outer $1 / 3$ and inner $2 / 3$ is explained in Figure E1 in more detail.

dye marks and microcoil(s) (Figure 1,C); 3-dimensional images were then built as reference for subsequent surgery (Figure 1,D). Alternatively, the procedures from bronchoscopic mapping through CT to surgery were conducted in a hybrid operation room in 1 session under general anesthesia.

\section{Surgery and Resection Margin Measurements}

Surgery was conducted the same day as marking or 1 or 2 days after. The surgical plan, constituting the approach (video-assisted thoracic surgery [VATS] or VATS-assisted mini-thoracotomy) and the resection method (wedge resection or segmentectomy) were registered preoperatively. Surgery was conducted under fluoroscopy to visualize the microcoils (Figure 1,E). Fluoroscopy was used 3 times: preoperatively to confirm the microcoil location; intraoperatively after applying a stapler but before firing the stapler to examine whether the tentative resection line determined by pleural lung mapping was appropriate (usually, this process was repeated each time a stapler was applied); and after completing resection to confirm that the microcoil was recovered inside the resected specimen. Resection margins, including the staple line, were measured macroscopically on the deflated lung specimen intraoperatively. The lesion diameter measured on preoperative CT was used to calculate the resection margins. If the resected lesion could not be identified macroscopically but was detected on microscopic or pathological examination, the margins were measured during pathological examination, considering the width of the staple line. The patients were followed for 30 days after the planned day of surgery. When adverse events occurred, patients were followed for more than 30 days.

\section{Primary End Point and Size Calculation}

The primary end point was successful resection, which was defined as lesion resection with resection margins larger than or equal to the lesion diameter or $2 \mathrm{~cm}$ using the initial planned resection. If additional resection was conducted, the resection margin measured at the initial resection was used to determine resection success. Resection was defined as unsuccessful regardless of the resection margins if insufficient lung mapping necessitated changes in the operation plan (eg, conversion from wedge resection to segmentectomy) or approach (eg, conversion from VATS to open thoracotomy, for palpation).
The details of determining the primary goal and sample size calculation were described previously. ${ }^{11}$ Most important, among patients in the previous study undergoing original VAL-MAP, the successful resection rate of those meeting the inclusion criteria in the present study was $78 \%$. Assuming the superiority of VAL-MAP 2.0 over the conventional method for resecting deeply located nodules with adequate margins, a successful resection rate of $80 \%$ was considered an appropriate primary goal. Accordingly, the required sample size was calculated as 60 lesions; considering a dropout rate of approximately $10 \%$, we set the target sample size at 65 lesions.

\section{Secondary End Points}

The secondary end points were (1) lung mapping effectiveness, including dye marking ${ }^{4}$ and microcoil placement success rates; (2) the effectiveness of mapping-assisted surgery assessed by changes in the surgical approach (thoracoscopy alone, small thoracotomy, or open thoracotomy) or resection method (wedge resection, segmentectomy, or lobectomy), and a survey of the surgeons' opinions regarding the contribution of VAL-MAP 2.0 to the surgery; and (3) safety. Microcoil placement success was evaluated according to whether the microcoils were placed at the planned positions assessed by post-mapping $\mathrm{CT}$ images and whether the microcoils remained in place until the end of resection, confirmed by intraoperative fluoroscopy. Locational differences within approximately $0.5 \mathrm{~cm}$ from the planned location were considered successful microcoil placements. Adverse events were recorded up to postoperative day 30 , and the cause and severity were assessed according to the definitions of The International Conference on Harmonization safety guideline E2a.

\section{Data Collection and Analysis}

All data recorded at each institution using an electronic data capture application service (Viedoc, Viedoc Technologies, Uppsala, Sweden). All data analyses were conducted independently by specialized statisticians in accordance with the study protocol. The patient characteristics data were expressed as median (interquartile range). For each primary and secondary end point, the success rates and $95 \%$ confidence intervals (CIs) were estimated. Operation time was expressed as median (interquartile range). Statistical analyses were performed using SAS 9.4 software (SAS Institute Inc, Cary, NC). 
Mapping design

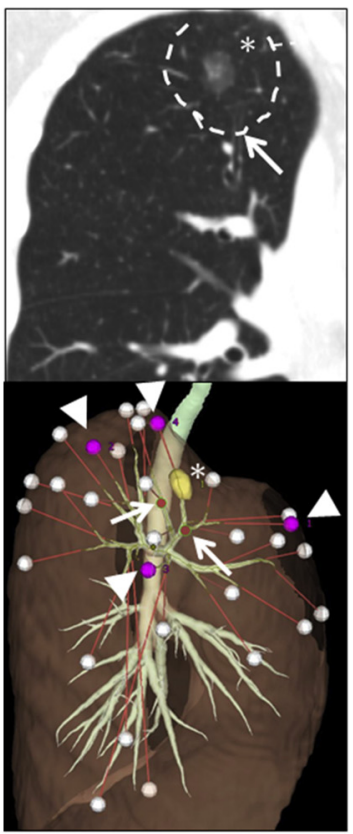

A

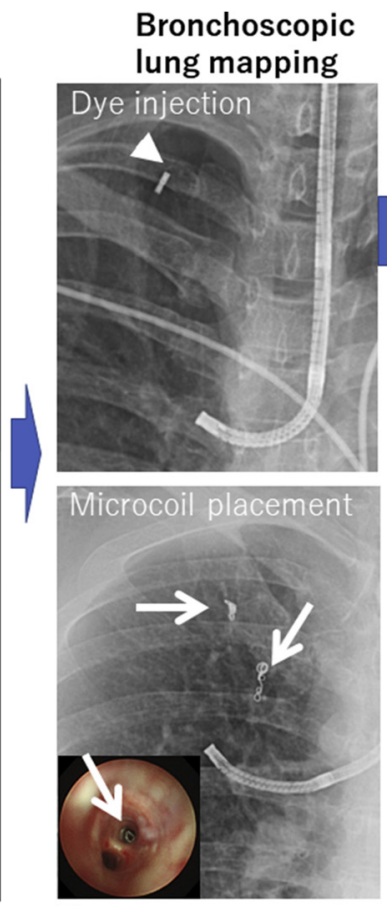

B
Post-mapping CT

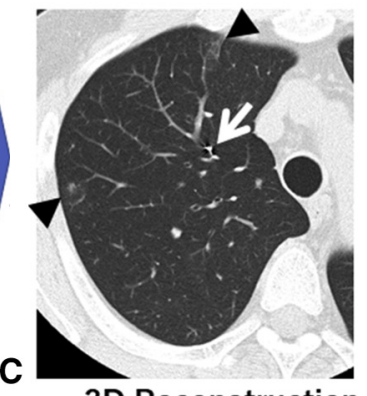

3D Reconstruction

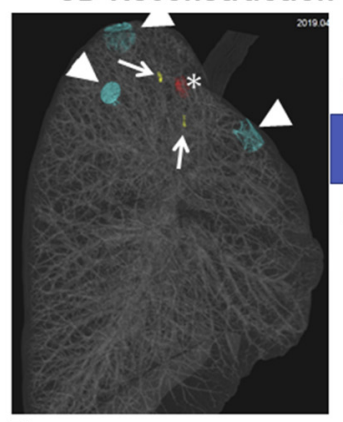

D
Operation

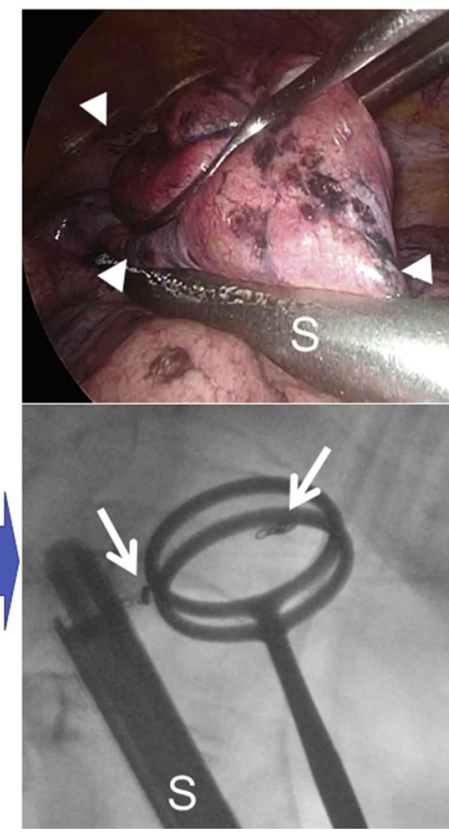

E

FIGURE 1. Steps in VAL-MAP 2.0. A, The 3-dimensional lung "map" was designed using CT images and a radiology workstation to develop virtual bronchoscopy. The interrupted line indicates the approximate resection line, considering the required resection margin. B, The bronchoscopic mapping procedure was performed within 48 hours before surgery under fluoroscopic guidance. Dye injection (upper) was followed by microcoil placement (lower). C, After mapping, CT was conducted within a few hours to visualize the actual locations of the marks. D, Using a radiology workstation, 3-dimensional (3D) images were further reconstructed, reflecting the actual mark locations. E, The operation was conducted using the 3D image for guidance. A fluoroscope was used to visualize the microcoils and guide deep resection lines at stapling (lower). The asterisks indicate the targeted nodules, and the arrowheads indicate dye marks or the planned locations of the dye marks. Arrows indicate microcoils or planned locations of microcoil placement, and "S" indicates a surgical stapler.

\section{Monitoring and Audit}

On-site monitoring of the quality and consistency of data collection was conducted at each institution, with additional central monitoring. An external audit was conducted at 2 representative institutions to review the appropriateness of the patients' records, electronic data capture, and

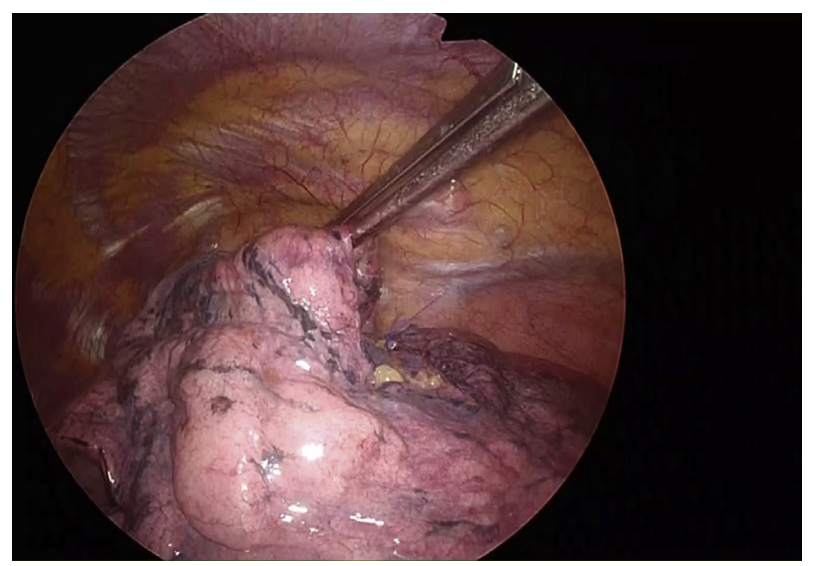

VIDEO 1. The background, methods including the technique of VALMAP 2.0, and results of the present study. Video available at: https:// www.jtcvs.org/article/S0022-5223(21)01365-9/fulltext. other related documents in accordance with the study protocol, followed by a closeout meeting and publication of an audit report.

\section{RESULTS \\ Patients}

Sixty-nine lesions from 65 cases (including patients registered twice for bilateral lesions) were initially registered. One patient with 1 lesion coughed out a microcoil immediately after placement; therefore, the microcoil did not remain in place until lung resection. This patient was excluded from the full analysis set when assessing the effectiveness of VAL-MAP 2.0. However, this patient was included in the safety analysis set and in the assessment of the microcoil placement success/failure. Two lesions in a patient who had 3 lesions to be resected, and 1 lesion in a patient who had 2 lesions to be resected were excluded from the VAL-MAP 2.0 effectiveness assessment because these lesions were not targeted by the technique; however, these lesions were included in the subsequent analyses. Thus, 65 lesions in 64 patients underwent planned surgery and were analyzed. The patient demographics 


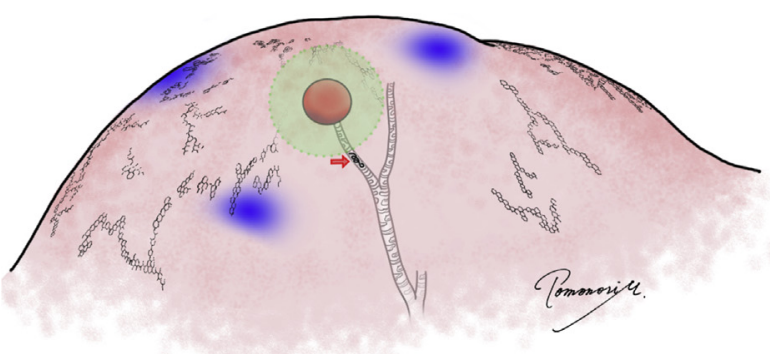

A

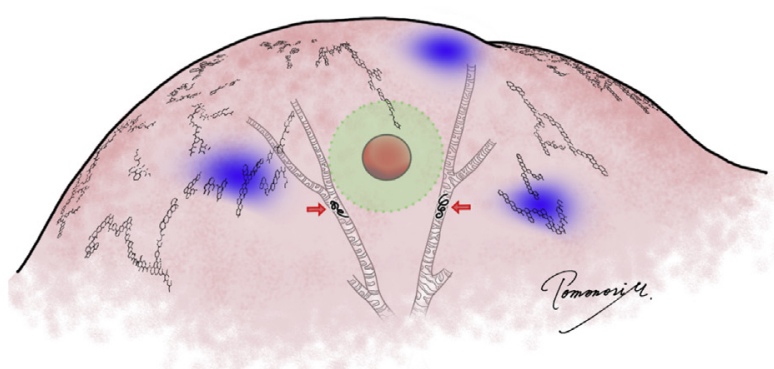

B

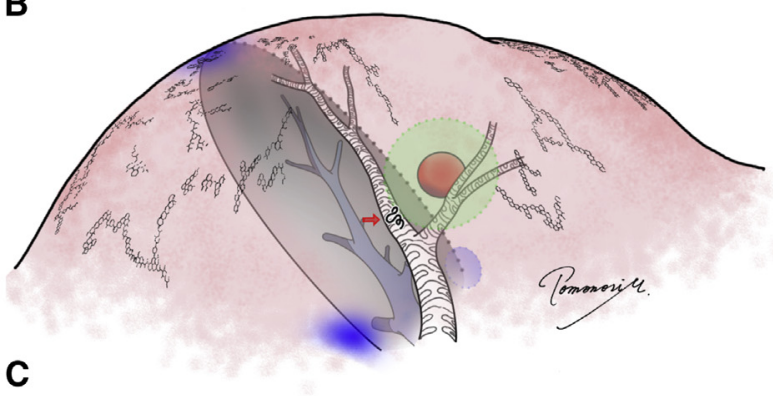

FIGURE 2. The strategy to plan the location of a microcoil in a bronchial root. A, In wedge resection, if a bronchus directly reached the target lesion or was near the target lesion, we selected the point of the bronchus central to the tumor to indicate the deep resection margin. B, Additionally, in wedge resection, if no bronchus directly reached the lesion, 2 bronchi reaching relatively close to the tumor were selected to indicate the deep resection margin. C, In segmentectomy, microcoil placement was designed to be a point on a bronchial tree indicating the most critical intrapulmonary resection margin (usually along the intersegmental plane).

of the full analysis set are shown in Table 2, and the characteristics of the targeted lesions are shown in Table 3. The CONSORT diagram is shown in Figure E2.

\section{Primary End Point}

Sixty-four of 65 lesions met the criteria for successful resection $(98.5 \%$; 95\% CI, 91.7-100; Figures 3 and 4, Video 1). The median resection margin was 20 (15-25) $\mathrm{mm}$. Without considering lung inflation/deflation, the median extra margin (ie, the difference between the measured resection margin on the specimen and the required resection margin measured on preoperative CT) was 8 (4-14) mm. Resection failure occurred for only 1 lesion $(1.5 \%)$, which had insufficient margins in wedge resection (Figure E3).
TABLE 2. Patient demographics of the full analysis set

\begin{tabular}{llc}
\hline & Median (interquartile range) \\
\hline Age $(\mathrm{y})$ & $66.5(56.5-75.0)$ \\
\hline Brinkman Index $(\mathrm{n}=28)$ & $490(190-1080)$ \\
\hline & Category & $\begin{array}{c}\text { No of } \\
\text { cases (\%) }\end{array}$ \\
\hline Sex & Male & $28(43.8)$ \\
History of primary lung cancer & Yes & $5(7.8)$ \\
History of malignancy other than lung cancer & Yes & $34(53.1)$ \\
Smoking history & Yes & $28(43.8)$ \\
\hline Present or past history of bronchial asthma & Yes & $1(1.6)$ \\
History of chest surgery on the same side & Yes & $5(7.8)$ \\
Timing of surgery after VAL-MAP & Same day & $16(25.0)$ \\
& $1 \mathrm{~d}$ after & $44(68.8)$ \\
& $2 \mathrm{~d}$ after & $4(6.3)$ \\
\hline
\end{tabular}

VAL-MAP, Virtual-assisted lung mapping.

\section{Secondary End Point: Effectiveness of Dye Marking}

Among 196 dye marks, 173 marks were successfully identified intraoperatively $(88.3 \%$; 95\% CI, 82.9-92.4). The grading of the dye marks is shown in Table E2. Most marks $(77.1 \%)$ were graded as appropriate (G2 and G3). The most common reason for a grading of G0 (invisible) was central injection, which caused technical marking failure in $52.2 \%$ of the marks (Table E3).

\section{Secondary End Point: Effectiveness of Microcoil Placement}

Among 75 microcoils placed, 64 were confirmed within $5 \mathrm{~mm}$ of the planned location in post-mapping CT; among them, $61(82.4 \%$; 95\% CI, 71.8-90.3) remained in place until the end of resection (successful placement) (Table 4). Among the 14 "unsuccessful" placements, 1 microcoil was displaced because of coughing and the microcoil was removed at that time; subsequent surgery was conducted without a microcoil. Ten microcoils were placed at locations away from the planned location by more than $5 \mathrm{~mm}$, mainly for technical or anatomic reasons, although the microcoils remained in place until the end of resection. Nine were recovered within the resected specimens, whereas the other microcoil required an additional small wedge resection for recovery. Two microcoils were stretched (ie, rolled inappropriately) to lodge in the airway during placement; 1 was found dislocated toward the pleura intraoperatively, and the other was found dislocated centrally and was recovered using a bronchoscope. Another microcoil was successfully placed at the planned position and remained in place until the beginning of resection, but was found centrally dislocated during resection because of intraoperative maneuvers. Notably, microcoil recovery by bronchoscopy intraoperatively $(\mathrm{n}=2)$ and the need for additional wedge resection $(n=1)$ were not considered 
TABLE 3. Characteristics of the targeted lesions

\begin{tabular}{llc}
\hline & \multicolumn{2}{c}{ Median (interquartile range) } \\
\hline Diameter (mm) & & $9(7-13)$ \\
Depth $(\mathrm{mm})$ & $11(5-15)$ \\
Required resection depth $(\mathrm{mm})^{*}$ & $30(25-35)$ \\
\hline & \multicolumn{1}{c}{ Category } & No of lesions (\%) \\
\hline CT characteristics & pure GGO & $21(32.3)$ \\
& GGO with solid component & $16(24.6)$ \\
& nodule/solid & $26(40.0)$ \\
& cavity & $2(3.1)$ \\
Clinical diagnosis & Primary lung cancer $\dagger$ & $40(61.5)$ \\
& Metastatic lung tumor & $24(36.9)$ \\
& Other & $1(1.5)$ \\
\hline
\end{tabular}

The number of lesions was 65. $C T$, Computed tomography; $G G O$, ground-glass opacity. *Required resection depth $(\mathrm{mm})=$ depth (distance from the closest pleura) $+\{$ diameter $\times 2$ (tumor $<2 \mathrm{~cm})]$ or $[$ diameter +20 (tumor $\geq 2 \mathrm{~cm}$ )] $\}$. $\dagger$ Including suspected diagnosis.

severe adverse events by the external safety evaluating committee because a bronchoscope was necessary during surgery regardless, and wedge resection was peripheral and minor. In 1 patient, 2 microcoils were successfully placed at the planned location; however, because of patient-related medical factors, the subsequent surgery was postponed by 1 month. During the 1-month period, the microcoils remained in place without additional problems.

\section{Secondary End Point: Effectiveness of Marking- Assisted Surgery}

Completely thoracoscopic surgery was performed in 59 cases $(92.2 \%)$, and minithoracotomy with the aid of thoracoscopy was performed in 5 cases $(7.8 \%)$, the latter of which was selected according to preoperative information, such as anticipated severe adhesions. There were no changes in the surgical approach. We conducted 65 resections, most commonly wedge resection (60 lesions; $92.3 \%$ ) and segmentectomy (5 lesions; $7.7 \%$ ). In 1 wedge resection, frozen section suggested a higher-grade malignancy than expected, and additional lobectomy was conducted during the same surgery. Because the resection margin of the initial wedge resection met the criteria for resection success, the resection was judged as successful. All other resections were performed as planned, and no cases were judged unsuccessful because of changes in the approach or resection methods. The median operation time among cases undergoing a single resection was 89 (66-146) minutes for wedge resection $(\mathrm{n}=57)$ and 230 (161-247) minutes for segmentectomy $(\mathrm{n}=5)$. The contribution of VAL-MAP to surgery as evaluated by each surgeon per case was most commonly graded as "impossible to achieve accurate resection without mapping" (50 cases; $78.1 \%$ ), followed by "possible to achieve

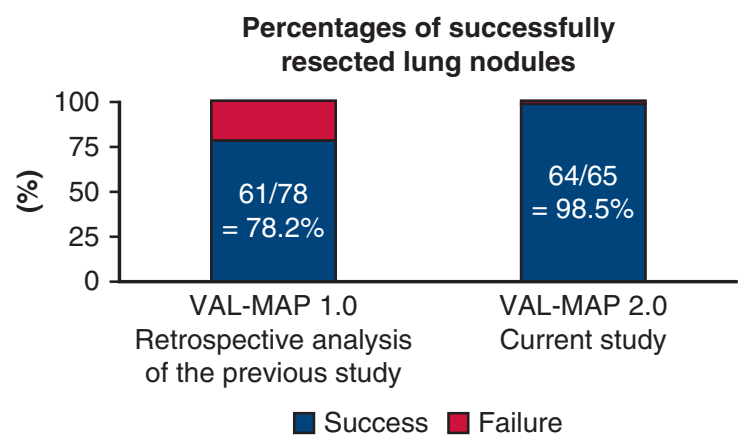

FIGURE 3. Resection success/failure with sufficient resection margins. Among patients in the previous study using the original VAL-MAP procedure or VAL-MAP $1.0,{ }^{4}$ the successful resection rate of those meeting the inclusion criteria in the present study was 61 of 78 resections $(78.2 \%$; left) and a successful resection rate of $80 \%$ was considered an appropriate primary goal indicating superiority of VAL-MAP 2.0 over VAL-MAP 1.0. Details are described in Materials and Methods. Using VAL-MAP 2.0 in the present study, resection success with sufficient resection margins (larger than or equal to the lesion diameter or $2 \mathrm{~cm}$ ) was achieved in 64 of 65 lesions ( $98.5 \%$; right).

the same operation, but mapping enabled confident resection" ( 14 cases; $21.9 \%$ ). No cases were judged as "mapping was unnecessary to complete the same resection."

\section{Secondary End Point: Safety}

From mapping to postoperative day 30, adverse events regardless of the association with VAL-MAP were observed in 26 of 65 patients $(40.0 \%$; Table E4). Adverse events during the bronchoscopic procedure were relatively minor, such as temporary hypertension, and none were associated with microcoil placement. Post-mapping CT revealed minor pneumothorax in 4 patients $(6.2 \%)$, and none required additional treatment. By the time of surgery, 4 patients $(6.2 \%)$ had experienced an adverse event, most frequently fever $38.0^{\circ} \mathrm{C}$ or greater (2 patients; $3.1 \%$ ). Postoperative adverse events were observed in 11 patients $(16.9 \%)$, most frequently prolonged air leakage lasting more than 6 days ( 3 patients; $4.6 \%$ ). Severe adverse events necessitating prolonged hospitalization were reported only postoperatively in 5 patients $(7.7 \%)$, namely, prolonged air leakage in 2 patients, followed by pneumonia, pleural effusion, and cholecystitis in 1 patient each. None of the adverse events were considered associated with the VAL-MAP 2.0 procedure, and no patients died.

\section{DISCUSSION}

VAL-MAP 2.0 was developed to overcome the limitations of conventional VAL-MAP, especially when acquiring deep resection margins in sublobar lung resection, ${ }^{4,10}$ In the present study, successful resection with adequate resection margins was achieved in 98.5\% of the cases, which was higher than the primary goal of $80 \%$. 
VAL-MAP 2.0 (dye + microcoil) secured deep resection margins in sublobar lung resections

\begin{tabular}{|c|}
\hline $\begin{array}{l}\text { Methods: A multi-center prospective single } \\
\text { arm study examining the effect of VAL-MAP } \\
2.0 \text { (dye marks + microcoils) on successful } \\
\text { resection of deep lung nodules with margins } \\
\geq \text { tumor diameter or } 2 \mathrm{~cm} \text {. }\end{array}$ \\
\hline$\downarrow$ \\
\hline $\begin{array}{l}\text { Main Results: Resection success was } \\
\text { achieved in } 64 / 65 \text { lesions ( } 98.5 \% \text { ), which is } \\
\text { higher than that expected from the previous } \\
\text { study using VAL-MAP } 1.0 \text { (dye marks only). } \\
\text { Safety of microcoils was also confirmed. }\end{array}$ \\
\hline$\downarrow$ \\
\hline $\begin{array}{l}\text { Take Home Message: VAL-MAP } 2.0 \text { can } \\
\text { facilitate successful resections for deep } \\
\text { pulmonary nodules, overcoming the } \\
\text { limitations of conventional VAL-MAP. }\end{array}$ \\
\hline
\end{tabular}

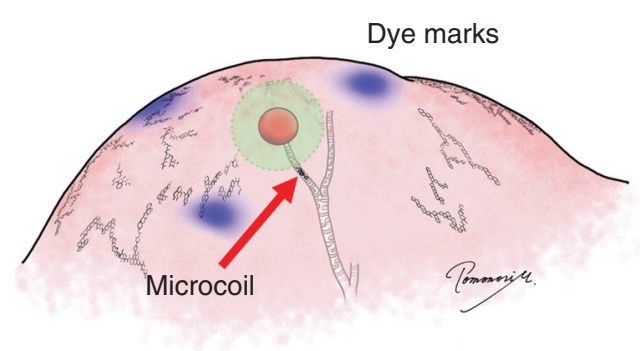

Resection success/failure of deep lung nodules

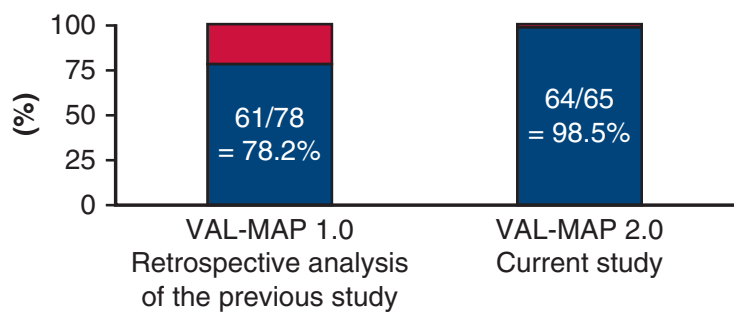

Success $\square$ Failure

FIGURE 4. VAL-MAP 2.0 (dye + microcoil) secured deep resection margins in sublobar lung resections.

Conventional VAL-MAP provides no information inside the lung. ${ }^{4}$ This limitation is common among localization techniques on the pleural surface, such as CT-guided markings, translated into insufficient margins in wedge resection of deep tumors. ${ }^{9}$ In the VAL-MAP 2.0 feasibility study, tentative staple lines set according to superficial dye marks required modification after visualizing microcoils on fluoroscopy in half of the cases. ${ }^{10}$ Therefore, dye marks alone are insufficient to set accurate deep resection lines, and another mark inside the lung (ie, a microcoil) is helpful. ${ }^{2}$ Supporting this hypothesis, the present study achieved high successful resection rates with sufficient margins of $98.5 \%$.
Although experience with VAL-MAP may have contributed to the outcome, considering the primary goal of the study of $80 \%$ set according to a previous study using conventional VAL-MAP, ${ }^{4}$ this result was unlikely without microcoils. Notably, although "successful" microcoil placement was achieved for 61 of 75 microcoils, the failed coils were still helpful intraoperatively. For example, although 10 microcoils were judged as failed because the location was more than $5 \mathrm{~mm}$ away from the planned location, most were still considered helpful as markers or reference points inside the lung, especially because the final location was confirmed by post-mapping CT.

TABLE 4. Details of microcoil placement

\begin{tabular}{|c|c|c|}
\hline Microcoil placement & Details & $\begin{array}{l}\text { No. of } \\
\text { microcoils }\end{array}$ \\
\hline \multirow{2}{*}{$\begin{array}{l}\text { Successful* } \\
\quad(\mathrm{n}=61)\end{array}$} & Successfully placed as planned and resected within the specimen & 59 \\
\hline & Operation postponed in 1 patient, and 2 microcoils remained in place for 1 mo until resection & 2 \\
\hline \multirow{6}{*}{$\begin{array}{l}\text { Unsuccessful } \\
\qquad(\mathrm{n}=14)\end{array}$} & Displaced because of coughing during bronchoscopy, and the microcoil was removed immediately & 1 \\
\hline & $\begin{array}{l}\text { Displaced } \geq 5 \mathrm{~mm} \text { at bronchoscopy, mostly for technical or anatomic reasons; remained in place } \\
\text { until the end of resection, and recovered within the specimen }\end{array}$ & 9 \\
\hline & Displaced into another segment at bronchoscopy and recovered by additional wedge resection & 1 \\
\hline & Stretched at placement and found dislocated toward the pleura during surgery. Recovered within the specimen. & 1 \\
\hline & Extended at placement and found dislocated centrally by surgery. Recovered using bronchoscopy during surgery. & 1 \\
\hline & $\begin{array}{l}\text { Placed as planned and remained in place until surgery, but centrally dislocated by intraoperative } \\
\text { manipulation and bronchoscopically recovered. }\end{array}$ & 1 \\
\hline
\end{tabular}

*Successful microcoil placement was defined as bronchoscopic placement within $5 \mathrm{~mm}$ of the planned location on CT. 
Given that additional microcoils increase the efficacy of deep lung resection, it is reasonable to question whether placing a microcoil alone is sufficient. Localization techniques using microcoils have been reported using CT-guided percutaneous placement ${ }^{13,14}$ or bronchoscopic placement. ${ }^{15}$ The former was also combined with the pleural dye mapping of original VAL-MAP, ${ }^{16}$ and the latter was applied in VAL-MAP 2.0. In VAL-MAP 2.0, both dye marks and microcoils can be placed bronchoscopically in 1 session. Furthermore, unlike the CT-guided percutaneous method, a microcoil can be placed even central to a deep pulmonary nodule to secure deep resection margins, avoiding the risk of air embolism. Regarding whether microcoils could completely replace dye marks, it is important to emphasize that lung "mapping" is a concept beyond simple localization, for which a microcoil alone might be enough. In VAL-MAP, the lung map not only indicates the tumor location, but navigates a stapler around the tumor to secure resection margins. For deep tumors, microcoils are critical to acquire deep resection margins, whereas the pleural dye marks play important roles in setting a tentative resection line to determine the stapler angles. Although neither dye marks nor microcoils were always placed perfectly, overall resection was highly successful and beyond the success of each method alone.

Given the technical limitations of dye marks and microcoil placement, there is room for improvement. First, an approximately $10 \%$ marking failure rate has been reported with dye marks using indigo carmine. ${ }^{4,5}$ Although technical issues were discussed in detail previously, ${ }^{17}$ the recent introduction of indocyanine green to VAL-MAP appears to have increased the visualization sensitivity. ${ }^{18}$ Regarding microcoils, an important finding of this study was the reliability of the microcoil we used, with no complications associated with placement, and the microcoils were generally stable in their locations until the end of resection (Table 4). Notably, most of the technical issues in microcoil placement occurred early in institutions that introduced VAL-MAP 2.0 for the first time. All participating centers had already introduced the original VAL-MAP and thus were familiar with virtual bronchoscopy and computerbased preoperative planning. The average time spent dye mapping with original VAL-MAP was $15.0 \pm 5.1$ minutes $(3.7 \pm 1.0 \mathrm{~min} / \mathrm{mark}),{ }^{6}$ and additional microcoil placement in VAL-MAP 2.0 required only a few minutes, although we did not record the details of the time required, in this study. However, additional hands-on learning could have steepened the learning curve.

\section{Study Limitations}

The present study has limitations. First, because this was a single-arm study, the results are not easily comparable with those of previous studies using original VAL-MAP and other localization techniques. In this study, we selected challenging deep lesions that could benefit from microcoil placement (criterion 3, Table 1). A randomized controlled trial using these criteria was considered unfair or even unethical in patients allocated to the control arm. Alternatively, we could have eliminated criterion 3 (Table 1); however, this would have required larger patient numbers to acquire sufficient statistical power because the original VAL-MAP technique is efficient for most shallow lesions. Second, the participating centers were mostly wellexperienced regarding VAL-MAP, and thus, intention to acquire sufficient resection margins was well fostered. In our previous study, we found high variability in resection success among participating centers despite relatively constant success in dye mapping, suggesting that surgeons' careful attention to resection margins is important. ${ }^{4}$ It is possible that the participation of expert centers biased this study. Third, resection margins were measured on deflated lung specimens, whereas targeted resection margins were determined in inflated lungs on CT images. However, even considering these potential biases and the fact that the margin measurement in deflated lung specimens was biased against resection success, we consider the excellent resection success is barely achievable with conventional VAL-MAP, and the contribution of microcoils was significant. Finally, selecting the operation type was not well balanced, and segmentectomy was selected in only 5 cases, likely because of the strict inclusion criteria for segmentectomy. A microcoil inside the lung in VAL-MAP 2.0 is likely to enlarge the application for wedge resection. Conversely, the role of VAL-MAP 2.0 in segmentectomy must be examined further, especially given the possibility that segmentectomy can be applied even more widely in lung cancer. $^{19}$

\section{CONCLUSIONS}

VAL-MAP 2.0, combining multi-spot dye marks on the lung and microcoils inside the lung, was highly helpful in achieving successful resection with adequate resection margins for deep pulmonary nodules, overcoming the limitations of conventional VAL-MAP.

\section{Conflict of Interest Statement}

The authors reported no conflicts of interest.

The Journal policy requires editors and reviewers to disclose conflicts of interest and to decline handling or reviewing manuscripts for which they may have a conflict of interest. The editors and reviewers of this article have no conflicts of interest.

The authors thank the staff from all the centers that participated in the present study, especially Drs Masahiro Yanagiya and Kazuhiro Nagayama (The University of Tokyo), Drs Ryo Wakejima, Yasuhiro Nakashima, and Kenichi Okubo (Tokyo Medical and Dental University), Drs Yuki Morimura and Shinji 
Kosaka (Shimane Prefectural Central Hospital), Dr Tomoki Nishida (Shonan Kamakura General Hospital), Drs Hiroaki Toba and Naoya Kawakita (Tokushima University), Dr Akihiro Taira (University of Occupational and Environmental Health), Drs Toru Bando and Atsushi Kitamura (St. Luke's International Hospital), and Dr Yoshinori Okada (Tohoku University). The authors thank the members of the clinical research support center at the University of Tokyo Hospital, especially Ai Okazaki (project manager), Dr Keiko Ueda (general manager), Drs Takuya Kawahara and Yukari Uemura (statistics), Yumi Tanaka (monitoring officers). The authors thank Jane Charbonneau, DVM, from Edanz Group (https://jp.edanz.com/ac), for editing a draft of this manuscript.

\section{References}

1. Sato M, Omasa M, Chen F, Sato T, Sonobe M, Bando T, et al. Use of virtual assisted lung mapping (VAL-MAP), a bronchoscopic multispot dye-marking technique using virtual images, for precise navigation of thoracoscopic sublobar lung resection. J Thorac Cardiovasc Surg. 2014;147:1813-9.

2. Sato M. Precise sublobar lung resection for small pulmonary nodules: localization and beyond. Gen Thorac Cardiovasc Surg. 2020;68:684-91.

3. Yanagiya M, Kawahara T, Ueda K, Yoshida D, Yamaguchi H, Sato M. A meta-analysis of preoperative bronchoscopic marking for pulmonary nodules. Eur J Cardiothorac Surg. 2020;58:40-50.

4. Sato M, Kobayashi M, Kojima F, Tanaka F, Yanagiya M, Kosaka S, et al. Effect of virtual-assisted lung mapping (VAL-MAP) in acquisition of surgical margins in sublobar lung resection. J Thorac Cardiovasc Surg. 2018;156:1691-701.

5. Sato M, Kuwata T, Yamanashi K, Kitamura A, Misawa K, Imashimizu K, et al. Safety and reproducibility of virtual-assisted lung mapping: a multicentre study in Japan. Eur J Cardiothorac Surg. 2017;51:861-8.

6. Sato M, Yamada T, Menju T, Aoyama A, Sato T, Chen F, et al. Virtual-assisted lung mapping: outcome of 100 consecutive cases in a single institute. Eur J Cardiothorac Surg. 2015;47:e131-9.

7. El-Sherif A, Fernando HC, Santos R, Pettiford B, Luketich JD, Close JM, et al. Margin and local recurrence after sublobar resection of non-small cell lung cancer. Ann Surg Oncol. 2007;14:2400-5.

8. Mohiuddin K, Haneuse S, Sofer T, Gill R, Jaklitsch MT, Colson YL, et al. Relationship between margin distance and local recurrence among patients undergo- ing wedge resection for small $(</=2 \mathrm{~cm})$ non-small cell lung cancer. $J$ Thorac Cardiovasc Surg. 2014;147:1169-75.

9. Yanagiya M, Sato M, Ueda K, Nagayama K, Kawahara T, Kawashima S, et al. Preoperative lung surface localization for pulmonary wedge resection: a single-center experience. J Thorac Dis. 2020;12:2129-36.

10. Sato M, Nagayama K, Kobayashi M, Nakajima J. Virtual-Assisted Lung Mapping 2.0: preoperative bronchoscopic three-dimensional lung mapping. Ann Thorac Surg. 2019;108:269-73.

11. Ueda K, Uemura Y, Sato M. Protocol for the VAL-MAP 2.0 trial: a multicentre, single-arm, phase III trial to evaluate the effectiveness of virtual-assisted lung mapping by bronchoscopic dye injection and microcoil implementation in patients with small pulmonary nodules in Japan. BMJ Open. 2019;9:e028018.

12. Sato M, Murayama T, Nakajima J. Concepts and techniques: how to determine and identify the appropriate target segment in anatomical pulmonary segmentectomy? J Thorac Dis. 2019;11:972-86.

13. Donahoe LL, Nguyen ET, Chung TB, Kha LC, Cypel M, Darling GE, et al. CTguided microcoil VATS resection of lung nodules: a single-centre experience and review of the literature. J Thorac Dis. 2016;8:1986-94.

14. Rodrigues JCL, Pierre AF, Hanneman K, Cabanero M, Kavanagh J, Waddell TK, et al. CT-guided microcoil pulmonary nodule localization prior to video-assisted thoracoscopic surgery: diagnostic utility and recurrence-free survival. Radiology. 2019;291:214-22.

15. Toba H, Kondo K, Miyoshi T, Kajiura K, Yoshida M, Kawakami Y, et al. Fluoroscopy-assisted thoracoscopic resection after computed tomography-guided bronchoscopic metallic coil marking for small peripheral pulmonary lesions Eur J Cardiothorac Surg. 2013;44:e126-32.

16. Yang SM, Lin CK, Chen LW, Chen YC, Huang HC, Ko HJ, et al. Combined virtual-assisted lung mapping (VAL-MAP) with CT-guided localization in thoracoscopic pulmonary segmentectomy. Asian J Surg. 2019;42:488-94.

17. Sato M. Virtual assisted lung mapping: navigational thoracoscopic lung resection. Cancer Res Front. 2016;2:85-104

18. Yanagiya M, Amano Y, Hiyama N, Matsumoto J. Initial experience of virtualassisted lung mapping utilizing both indocyanine green and indigo carmine. Gen Thorac Cardiovasc Surg. 2021;69:1035-9.

19. Suzuki K, Saji H, Aokage K, Watanabe SI, Okada M, Mizusawa J, et al. Comparison of pulmonary segmentectomy and lobectomy: safety results of a randomized trial. J Thorac Cardiovasc Surg. 2019;158:895-907.

Key Words: localization, lung cancer, margin, metastatic lung tumor, segmentectomy, wedge resection 

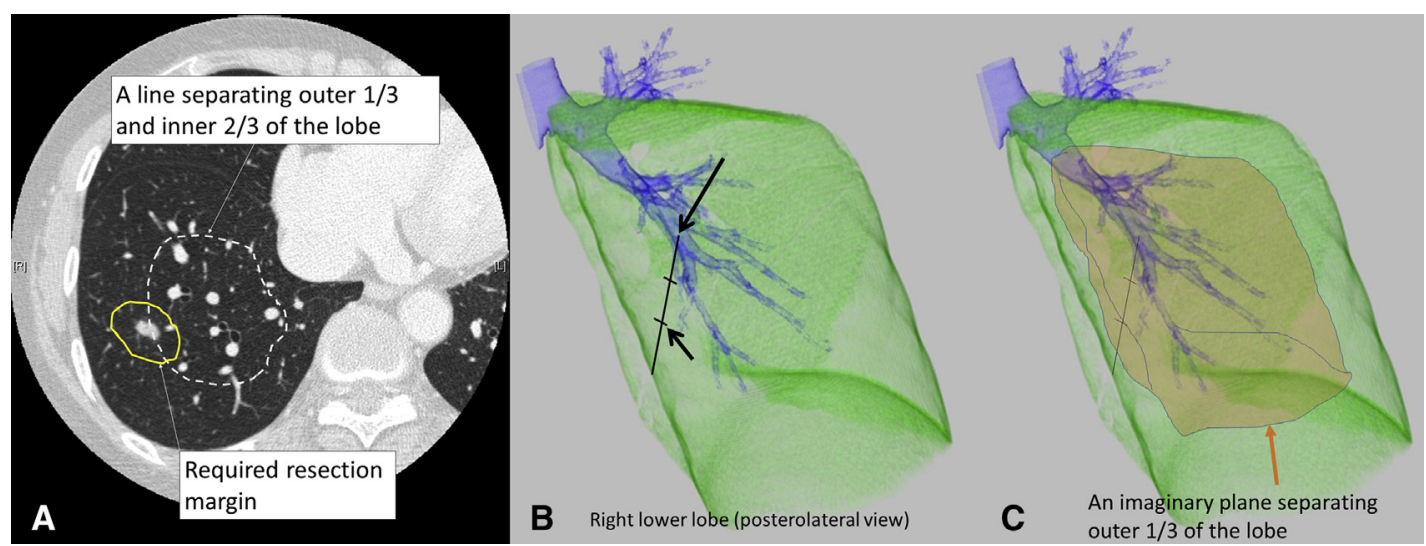

FIGURE E1. Visual explanation of the inclusion criteria, (3)-A and (3)-B. A, The interrupted line indicates an approximate line separating the outer 1/3 and inner $2 / 3$ of the pulmonary lobe on a CT image. Conceptually, this line is determined in a 3-dimensional model of the lung (B) as follows: The center of the lobe is defined as the hilum of the anatomical pulmonary segment where the targeted lesion is located, especially the insertion of the segmental bronchus into the lung parenchyma (long arrow in B). An imaginary line is drawn between the center and an arbitrary point on the pleural surface including the interlobar fissure, or the estimated interlobar fissure, if there is no lobulation. A point on the outer 1/3 line (short arrow in B) is selected for all possible lines between the lobe center and pleural surface and connected throughout the pulmonary lobe, developing an imaginary 3-dimensional plane separating the outer 1/3 and inner 2/3 of the lobe (C). The cutting surface of the plane on an arbitrary CT view (eg, an axial view including the lesion, as shown in A) translates into a line separating the outer $1 / 3$ and inner $2 / 3$ of the pulmonary lobe. Practically, recognizing this concept, surgeons draw an arbitrary line on CT images, not limited to the axial views but also using other views (eg, sagittal or coronal) if necessary, to evaluate whether the targeted lesion can be included in the study. 

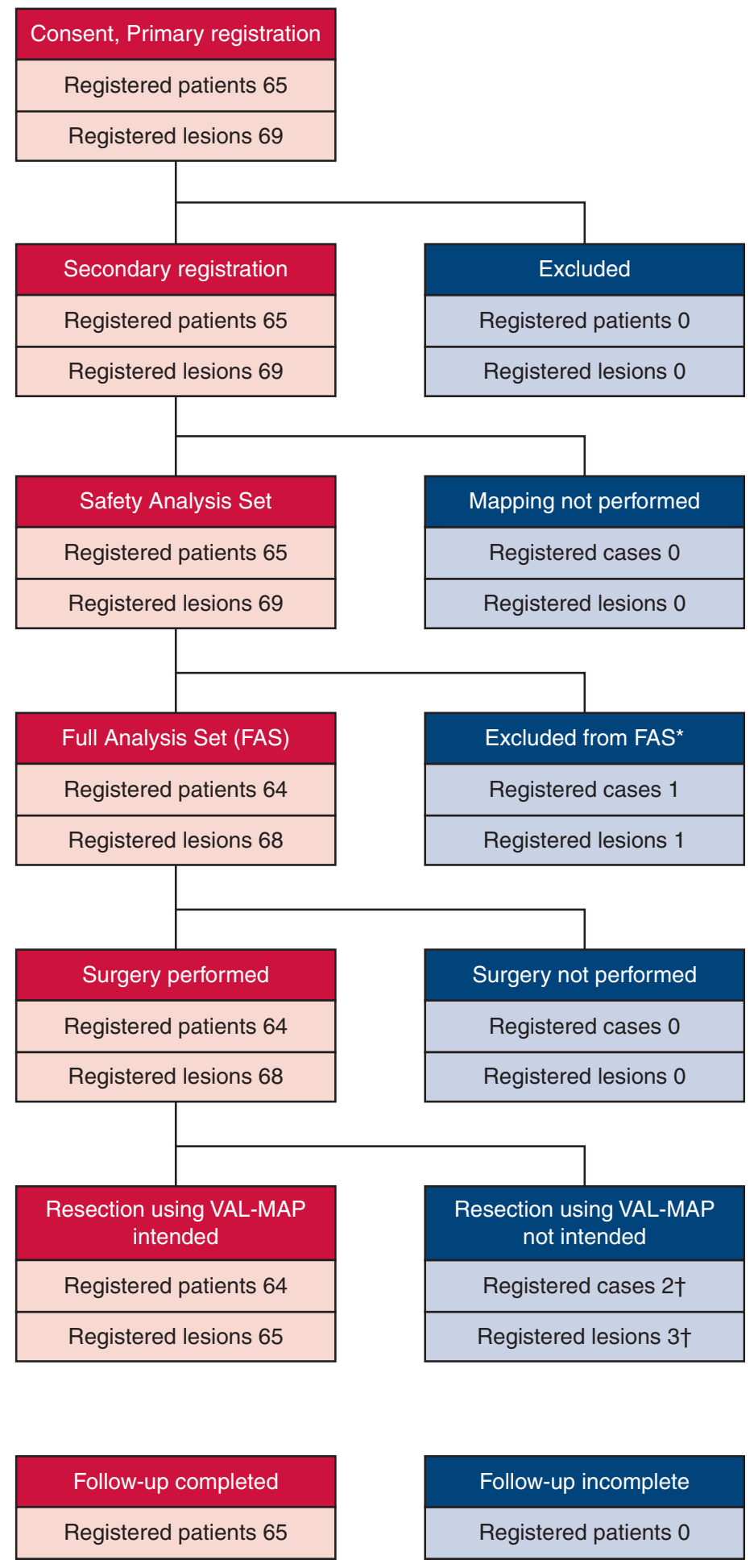

Follow-up incomplete

Registered patients 0

FIGURE E2. CONSORT diagram. *The reasons for exclusion from the full analysis set was migration and removal of the microcoil because of coughing during bronchoscopy, which made later evaluation of resection success using VAL-MAP 2.0 impossible. However, this patient was included in the safety analysis set and in the assessment of microcoil placement success/failure. †Three lesions in 2 patients were not targeted by VAL-MAP 2.0 ; however, these lesions were resected at the time of surgery. 


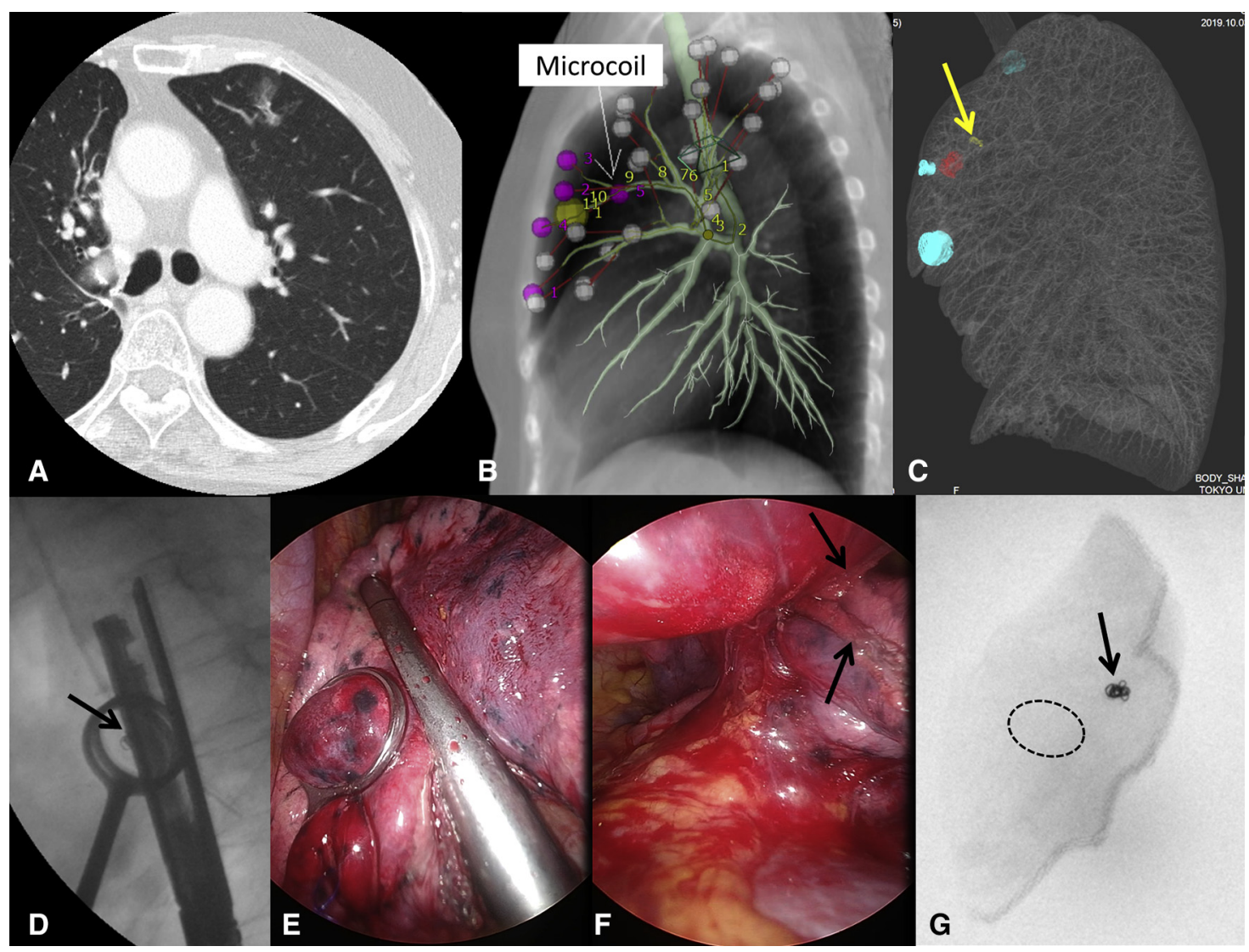

FIGURE E3. Details of the failed case. A, CT image showing a 20-mm diameter ground-glass nodule located in segment $\mathrm{S} 1+2$ of the left upper lobe. B, Preoperative mapping plan. The arrow indicates the location of the microcoil placement. The yellow, white, and purple dots indicate the tumor, candidate mapping points, and selected mapping points, respectively, on Synapse Vincent. C, Post-mapping 3D image showing a microcoil placed at the planned location (arrow), the tumor (red), and 3 dye marks (blue). D, Intraoperative fluoroscopic view taken when the stapler was applied. A microcoil is visible (arrow) within the ring forceps. E, Thoracoscopic view corresponding to (D). F, Thoracoscopic view from the mediastinal side after firing the stapler in (E). The arrows indicate the staple line relatively close to the hilum. The surgeon considered that the stapler could not be moved further toward the hilum and that the resection margin would be sufficient, according to the fluoroscopic view. G, Fluoroscopic view of the specimen, including the microcoil (arrow). The tumor location is shown as an interrupted circle. The measured resection margin in the deflated specimen was $15 \mathrm{~mm}$, which was smaller than the tumor diameter of $20 \mathrm{~mm}$ measured on preoperative CT, and in accordance with the definition of resection success in this study, the case was judged as resection failure. However, clinically, the resection margin was considered sufficient, and no additional resection was performed. 
TABLE E1. Steps in virtual-assisted lung mapping and the required equipment

\begin{tabular}{|c|c|c|c|c|}
\hline Steps & VAL-MAP 1.0 & VAL-MAP 2.0 & Items required & Recommendation \\
\hline Mapping design & レ & レ & Radiology work station & $\begin{array}{l}\text { Synapse Vincent ver. } 4 \text { or higher } \\
\text { (Fujifilm Medical, Tokyo, } \\
\text { Japan) }\end{array}$ \\
\hline \multirow[t]{6}{*}{$\begin{array}{l}\text { Bronchoscopic } \\
\text { lung mapping }\end{array}$} & $レ$ & 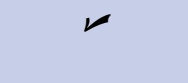 & Bronchoscope & $\begin{array}{l}\text { BF-P260 or P290 (Olympus, } \\
\text { Tokyo, Japan) }\end{array}$ \\
\hline & $レ$ & $\swarrow$ & Fluoroscope or cone-beam $\mathrm{CT}^{*}$ & \\
\hline & $\nu$ & $\nu$ & Dye-injection catheter & $\begin{array}{l}\text { P6-CW-1 (Olympus, Tokyo, } \\
\text { Japan) }\end{array}$ \\
\hline & & レ & Microcoil & $\begin{array}{l}\text { C-stopper (final diameter, } 5 \mathrm{~mm} \text {; } \\
\text { Piolax Medical Devices, } \\
\text { Tokyo, Japan) }\end{array}$ \\
\hline & & 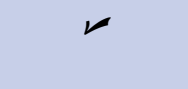 & Delivery microcatheter & $\begin{array}{l}\text { SIRABE (MC18-S110; Piolax } \\
\text { Medical Devices) }\end{array}$ \\
\hline & & $\boldsymbol{V}$ & Pusher & $\begin{array}{l}\text { C-stopper coil pusher (Piolax } \\
\text { Medical Devices) }\end{array}$ \\
\hline Post-mapping CT & レ & レ & Regular CT or cone-beam CT* & \\
\hline $3 \mathrm{D}$ reconstruction & $レ$ & $\swarrow$ & Radiology work station & $\begin{array}{l}\text { Synapse Vincent (any version; } \\
\text { Fujifilm Medical) }\end{array}$ \\
\hline Operation & レ & $\nu$ & Regular or hybrid* operation ro & \\
\hline
\end{tabular}

VAL-MAP, Virtual-assisted lung mapping; $C T$, computed tomography; 3D, 3-dimensional. *In a hybrid operation room equipped with a cone-beam CT.

TABLE E2. Intraoperative findings of dye marks

\begin{tabular}{lccc}
\hline \multicolumn{1}{c}{ Grades } & No. of marks & $(\%)$ \\
\hline G0: Unidentifiable & 23 & $(11.7)$ \\
G1: Identifiable, but faint and hardly visible & 14 & $(7.1)$ \\
G2: Easily identifiable without a central red spot or target-like shape & 116 & $(59.2)$ \\
G3: Easily identifiable with a central red spot & 35 & $(17.9)$ \\
G4: Target-like appearance with or without a central red spot & 1 & $(0.5)$ \\
G5: Bulla formation & 7 & $(3.6)$ \\
Total & 196 & $(100.0)$ \\
\hline
\end{tabular}


TABLE E3. Estimated causes of unidentifiable dye marks

\begin{tabular}{lcc}
\hline \multicolumn{1}{c}{ Estimated causes of invisible dye marks } & No. of marks & $(\%)$ \\
\hline Central injection & 12 & $(52.2)$ \\
\hline Anthracosis & 3 & $(13.0)$ \\
Emphysematous lung & 0 & $(0.0)$ \\
Pleural thickening & 6 & $(26.1)$ \\
Fading due to time lapse & 0 & $(0.0)$ \\
Insufficient injection force & 1 & $(4.3)$ \\
Catheter not reaching pleura due to intrapulmonary structure & 2 & $(8.7)$ \\
Overlapped marks & 1 & \\
Unknown & 0 & $(4.3)$ \\
Others & 3 & $(0.0)$ \\
Total & 28 & $(13.0)$ \\
\hline
\end{tabular}

The number of unidentifiable G0 marks was 23. Multiple causes could be selected for 1 mark.

TABLE E4. Reported adverse events throughout the study $(n=65)$

\begin{tabular}{|c|c|c|c|}
\hline Timing & Adverse events & No. of patients & $(\%)$ \\
\hline \multirow[t]{5}{*}{ During bronchoscopy } & Hypertension (systolic pressure $>180 \mathrm{~mm} \mathrm{Hg}$ for $>5 \mathrm{~min}$ or systolic pressure $>200 \mathrm{~mm} \mathrm{Hg}$ & 4 & $(6.2)$ \\
\hline & Hypotension (systolic pressure $<80 \mathrm{~mm} \mathrm{Hg}$ for $>5 \mathrm{~min}$ or systolic pressure $<60 \mathrm{~mm} \mathrm{Hg}$ & 1 & $(1.5)$ \\
\hline & Arrhythmia (including tachycardia $>140 \mathrm{bpm}$ or bradycardia $<50 \mathrm{bpm}$ & 1 & $(1.5)$ \\
\hline & Hypoxia ( $\mathrm{SpO} 2<90 \%$ for $>5 \mathrm{~min}$ or $\mathrm{SpO} 2<80 \%$ ) & 2 & $(3.1)$ \\
\hline & Severe cough (necessitating procedure interruption) & 2 & $(3.1)$ \\
\hline \multirow[t]{4}{*}{ Findings in post-mapping CT } & Minor pneumothorax (no need for treatment) & 4 & $(6.2)$ \\
\hline & Bulla formation & 4 & $(6.2)$ \\
\hline & Pneumomediastinum & 1 & $(1.5)$ \\
\hline & Chronic subdural hematoma* & 1 & $(1.5)$ \\
\hline \multirow[t]{3}{*}{ After mapping to surgery } & Fever $\left(\geq 38^{\circ} \mathrm{C}\right)$ & 2 & $(3.1)$ \\
\hline & Hypoglycemia, nausea & 1 & $(1.5)$ \\
\hline & Nausea & 1 & $(1.5)$ \\
\hline \multirow[t]{2}{*}{ Findings during surgery } & Subpleural hematoma & 1 & $(1.5)$ \\
\hline & pressure ulcer & 1 & $(1.5)$ \\
\hline \multirow[t]{8}{*}{ Postoperative events } & Pleural fistula/prolonged air leak $>6 \mathrm{~d} \dagger$ & 3 & $(4.6)$ \\
\hline & Atelectasis & 2 & $(3.1)$ \\
\hline & Pneumonia $\dagger$ & 1 & $(1.5)$ \\
\hline & Wound infection & 2 & $(3.1)$ \\
\hline & Pleural effusion $\dagger$ & 1 & $(1.5)$ \\
\hline & Ileus & 1 & $(1.5)$ \\
\hline & Cholecystitis $\dagger$ & 1 & $(1.5)$ \\
\hline & Subcutaneous emphysema & 1 & $(1.5)$ \\
\hline
\end{tabular}

bpm, Beats per minute; $C T$, computed tomography. *Clinically suspected and confirmed by brain CT taken concurrently with post-mapping chest CT. $\dagger$ Reported as severe adverse events due to prolonged hospitalization. 